\title{
Percepção do estresse e estressores de enfermeiros de um hospital universitário
}

\section{Perception of stress and stressors in nurses of a university hospital}

\section{Percepción de estrés y estresores en enfermeros de un hospital universitario}

\author{
J éssica Martins da Silva* \\ Universidade Federal do Rio de Janeiro - UFRJ, Rio de Janeiro, Rio de Janeiro, Brasil
}

Lucia Emmanoel Novaes Malagris**

Universidade Federal do Rio de J aneiro - UFRJ, Rio de Janeiro, Rio de Janeiro, Brasil

\begin{abstract}
RESUMO
Este estudo avaliou a percepção de estresse e estressores de enfermeiros de um hospital universitário e coletou sugestões de ações para amenizar o estresse. O estudo, descritivo, transversal, qualitativo e quantitativo foi realizado com 70 enfermeiros de diversas unidades de um hospital. Utilizouse a Escala de Estresse de Bianchi e uma pergunta aberta. Os dados quantitativos foram analisados pelo programa SPSS e foram feitas análises descritivas e inferenciais. Para os dados qualitativos, utilizou-se a técnica de análise de conteúdo de Bardin. Resultados indicaram que a maior parte (67\%) dos sujeitos apresentou nível médio de estresse. O domínio B (atividades relacionadas ao funcionamento adequado da unidade) teve a média de escore de estresse mais alta $(5,2)$ em todas as unidades estudadas. A média de estresse dos enfermeiros que exercem função de chefia foi significantemente maior do que a média dos que não exercem $(p=0,002)$. A área temática "Melhores condições para o trabalho" foi a mais citada como sugestão para amenizar o estresse. Os dados obtidos indicaram que a situação atual de precarização do hospital está relacionada com o estresse dos enfermeiros. Considera-se que o estudo permitiu ampla compreensão sobre o estresse e estressores em enfermeiros do hospital e subsídios para estudos posteriores.
\end{abstract}

Palavras-chave: estresse, estressores, enfermeiros, hospital universitário.

\begin{abstract}
This study evaluated the perception of stress and stressors of nurses at a university hospital and collected suggestions for actions to relieve stress. Descriptive, cross-sectional qualitative and quantitative study with 70 nurses from different hospital units. The Bianchi Stress Scale was used and an open question was applied. The quantitative data were analyzed using the SPSS program and descriptive and inferential analyzes were used. For qualitative data, the Bardin content analysis technique was used. The results indicated that most of the participants $(67 \%)$ had a medium level of stress. Domain B
\end{abstract}


(activities related to the proper functioning of the unit) had the highest average stress score in all units studied. The mean stress level of nurses who performed leadership was significantly higher than the average of those who did not $(p=0.002)$. The thematic area "Better conditions for work" was the most cited as a suggestion to relieve stress. The data obtained indicates that the current situation of precariousness of the hospital is related to the nurses' stress. It is considered that the study allowed a broad understanding on the stress and stressors of hospital nurses and subsidies for further studies.

Keywords: stress, stressors, nurses, universitary hospital.

\section{RESUMEN}

Este estudio evaluó la percepción del estrés y estresores de enfermeros de un hospital universitario y recogió sugerencias de acciones para amenizar el estrés. Estudio descriptivo, transversal, cualitativo y cuantitativo se realizó con 70 enfermeros de varias unidades de un hospital. Se utilizó la Escala de estrés de Bianchi y una pregunta abierta. Los datos cuantitativos fueron analizados por el programa SPSS. Para los datos cualitativos se utilizó la técnica de análisis de contenido de Bardin. Los resultados indicaron que $67 \%$ presentaron un nivel medio de estrés. El dominio B (actividades relacionadas al funcionamiento adecuado de la unidad) tuvo el promedio de puntuación de estrés más alta $(5,2)$ en todas las unidades estudiadas. El promedio de estrés de los enfermeros que ejercen función de dirección fue significativamente mayor que el promedio de los que no ejercen (Test $t$ de student $p=0,002)$. El área temática "Mejores condiciones para el trabajo" fue la más citada como sugerencia para amenizar el estrés. Los datos obtenidos indican que la situación de precarización del hospital está relacionada con el estrés de los enfermeros. Se considera que el estudio permitió una amplia comprensión sobre el estrés y estrés de los enfermeros del hospital y subsidios para estudios posteriores.

Palabras clave: estrés, estresores, enfermeros, hospital universitario.

Estudos apontam que, embora o estresse surja em diversas profissões, o profissional da saúde faz parte de uma das classes de trabalho mais afetadas pelo adoecimento gerado por essa condição. Esse dado deve-se ao fato de que no ambiente laboral, onde este tipo de profissional atua, existem vários estressores, tais como sobrecarga de trabalho, interrupções frequentes, realização de tarefas simultâneas, entre outros (Puerto, Soler, Montesinos, Marcos, \& Chorda, 2017).

A International Labour Organization (ILO) realizou, em 2016, uma pesquisa com especialistas de 54 países, obtendo como resultado a indicação de $90 \%$ dos participantes de que o estresse é um problema global. Dessa forma, os riscos psicossociais do estresse expandiram o interesse de pesquisadores da Psicologia e de demais áreas para pesquisas e políticas sobre o tema (ILO, 2016).

Hans Selye, físico e endocrinologista, foi o precursor do estudo sobre o estresse e o definiu como o resultado inespecífico de alguma demanda sobre o corpo (ILO, 2016). De modo geral, as definições de estresse envolvem a relação entre o indivíduo e o ambiente. Pode ser 
entendido como um estado de desequilíbrio do funcionamento diante de situações desafiantes. Esses eventos levam o organismo a utilizar seus recursos psicobiológicos a fim de recuperar o equilíbrio (Lipp, 2017). O impacto do estresse na saúde pode variar de acordo com a resposta individual. Entretanto, altos níveis de estresse podem gerar enfermidades físicas como doenças cardiovasculares e transtornos mentais, tais como burnout, ansiedade e depressão (ILO, 2016).

Com base em pesquisas teóricas e empíricas, entende-se que a profissão de enfermeiro tem alta relação com o estresse. Cuidar do enfermeiro, portanto, destaca-se como elemento fundamental. Diante disso, a Psicologia da Saúde vem se destacando em âmbitos multidisciplinares e interdisciplinares para a compreensão dos elementos envolvidos na questão da saúde e do estresse ocupacional desses profissionais (Xavier, Reis, \& Frassão, 2016).

O estresse ocupacional pode ser conceituado como um processo no qual o indivíduo percebe estressores no ambiente do trabalho sobre os quais, ao exercer sua habilidade de enfrentamento, provocam no sujeito reações negativas (Paschoal \& Tamayo, 2004). Alguns estudos indicaram a Síndrome de Burnout (SB) como condição desencadeada pelo estresse ocupacional, resultante da tensão crônica no trabalho (Moraes Filho \& Almeida, 2016; Oliveira et al., 2017). Embora a relação entre o estresse ocupacional e a SB seja amplamente reconhecida, neste estudo será analisado o estresse de forma mais ampla, ao buscar avaliar o nível de estresse e os estressores percebidos pelos enfermeiros.

Estressor pode ser definido como um estímulo capaz de eliciar as reações de estresse, ou seja, é tudo o que gera o estresse (Selye, 1965). Alguns autores entendem que os estressores por si só não geram o estresse. Tais teóricos defendem a ideia de que a interpretação que o indivíduo faz a respeito do estressor é fator condicionante do estresse. Assim, a forma como o enfermeiro utiliza as atividades cognitivas para entender os eventos no ambiente hospitalar é fundamental para o desencadeamento ou não do estresse (Lipp, 2017).

O ambiente hospitalar é considerado por diversos autores como estressante para os profissionais da saúde. Esse fato deve-se aos estressores que são vivenciados nesse local, como sofrimento, condições de trabalho, demandas de assistência e alta responsabilidade (Guido, Linch, Pitthan, \& Umann, 2011). Deve-se atentar para o fato de que o hospital é dividido em unidades que possuem as suas particularidades e acredita-se que essa questão pode influenciar na percepção do profissional a respeito do estresse e dos estressores.

Pesquisas indicam que a enfermagem está entre as profissões de saúde mais afetadas pelo estresse. Investigações recentes sobre o estresse em enfermeiros mostraram que mais da metade da amostra 
estudada apresentou nível de médio a alto de estresse (Almeida, A.K. Lima, Vasconcelos, A.C. Lima, Oliveira, 2016; Kirhhof, Oshôa, Bublitz, Lopes, \& Squiavenato, 2016; Soratto et al., 2016). De acordo com a literatura, os estressores associados à profissão do enfermeiro estão relacionados às demandas de trabalho, à pressão emocional, ao reconhecimento profissional e ao relacionamento interpessoal (Ueno et al., 2017).

Destaca-se que as profundas transformações dos hospitais nos últimos tempos alteraram a rotina de trabalho dos enfermeiros. A cultura do protocolo e o desenvolvimento da tecnologia fizeram com que o enfermeiro assumisse mais uma responsabilidade que é a de gestão de equipe (Rodrigues, Barrichello, \& Morin, 2016). Outro fator que deve ser considerado é a especificidade do trabalho do enfermeiro em hospital público, onde há déficit de recursos humanos e materiais. Tais déficits influenciam negativamente a qualidade da assistência e conflita com a idealização formada durante a graduação, o que gera estresse e sentimento de impotência (M. Mauro, Paz, C. Mauro, Pinheiro, \& Silva, 2010).

Destaca-se que o campo de pesquisa deste estudo foi um hospital universitário (HU). De acordo com o Ministério da Educação (MEC), HUs são centros de formação de recursos humanos e de desenvolvimento de tecnologia para a área de saúde (Brasil, n.d.). O contexto desse tipo de hospital apresenta algumas especificidades que são consideradas agravantes para o estresse: a precarização dos recursos físicos, materiais e humanos e a responsabilidade de assessoramento nas atividades de ensino, pesquisa e extensão (Médici, 2001).

\section{Método}

Trata-se de um estudo descritivo, transversal, qualitativo e quantitativo com enfermeiros de diversas unidades do Hospital Universitário Clementino Fraga Filho (HUCFF/UFRJ).

\section{Participantes}

Foram selecionados para o estudo todos os enfermeiros efetivos do HUCFF/UFRJ das seguintes unidades: ambulatórios (12 enfermeiros), clínica médica (17), centro cirúrgico (14), CTI (16), emergência (10), hematologia/transplante (12), nefrologia/hemodiálise (13) e UTI cardíaco (14), que totalizaram 108 enfermeiros (51\% do total de enfermeiros do hospital). Essas unidades foram selecionadas pela maior facilidade de comparação entre as mesmas devido ao número similar de profissionais. Foram excluídos os enfermeiros que no período da pesquisa estavam de licença (de qualquer tipo), os que 
tinham menos de 12 meses de atuação na unidade, além dos enfermeiros temporários e extraquadros. Dos 108 enfermeiros, 11 estavam de licença, 3 haviam se aposentado, 1 tinha menos de 12 meses de trabalho na unidade, 2 foram realocados para outras unidades e com 21 enfermeiros a pesquisadora não conseguiu contato para entregar/coletar o questionário. Desta forma, a amostra final foi constituída por 70 enfermeiros das seguintes unidades: Ambulatório (7), Centro Cirúrgico (10), Clínica Médica (11), CTI (11), Emergência (3), Nefrologia e Hemodiálise (9) e Hematologia e Transplante (8).

\section{Instrumento}

A Escala Bianchi de Stress busca medir o nível de estresse que o enfermeiro da área hospitalar atribui à atividade desempenhada. É uma escala autoaplicável, do tipo Likert, com 51 itens divididos em seis domínios para conhecimento das áreas de maior estresse: A: relacionamento (itens 40 a 46, 50 e 51); B: funcionamento adequado da unidade ( 1 a 6); C: administração de pessoal ( 7 a 9 e 12 a 14); D: assistência de enfermagem (16 a 30); E: coordenação da unidade $(10,11,15,31,32,38,39$ e 47) e F: condições de trabalho (33 a 37, 48 e 49). O nível de estresse do enfermeiro é avaliado pelo total de pontos da escala: de 51 a 357 pontos. Para obter o escore médio de cada domínio (variação de 1,0 a 7,0) somou-se os escores dos itens de cada domínio e dividiu-se pelo número de itens. Sendo: igual ou abaixo de 3,0: baixo nível de estresse; entre 3,1 a 5,9: médio nível de estresse; igual ou acima de 6,0: alto nível de estresse. Para se obter o escore médio para um determinado grupo, dividiu-se o total real do estressor pelo número de respondentes que assinalaram valores diferentes de 0 no item. O valor resultante foi a média real para cada estressor (item). Os escores médios dos 51 itens foram comparados entre eles, obtendo-se o estressor de maior intensidade para o grupo (Bianchi, 2009).

\section{Coleta de Dados}

Após a autorização da Direção da Enfermagem, da Direção do Hospital e do Comitê de Ética em Pesquisa (Parecer no2201303), a pesquisadora entrou em contato com as chefias de enfermagem de cada unidade para informar sobre a pesquisa e decidir o melhor horário de administração do instrumento. Os enfermeiros que aceitaram participar da pesquisa foram informados sobre o seu objetivo e procedimentos e a seguir, instruídos a preencherem 0 Termo de Consentimento Livre e Esclarecido (TCLE), a responderem à escala e à pergunta aberta: "Quais ações você acha que poderiam amenizar o estresse do enfermeiro?" 


\section{Análise de Dados}

Os dados foram analisados no programa SPSS (Statistical Package for the Social Sciences). Foram feitas análises descritivas e inferenciais. O teste de t-student foi usado para comparar dois grupos independentes e o Teste Análise de Variância (ANOVA) para comparar mais que dois grupos independentes e dependentes. Para a correlação entre variáveis foi calculado o coeficiente de correlação $r$ de Pearson. Os resultados foram considerados estatisticamente significantes se $p<0,05$, com intervalo de $95 \%$ de confiança.

Para análise da pergunta aberta, foi utilizada a técnica de análise de conteúdo de Bardin (2011), que analisa o conteúdo de entrevistas. Procura-se nesta técnica classificar o conteúdo em temas/categorias para a compreensão do que vai além do discurso (Silva \& Fossá, 2017).

\section{Resultados}

Ao avaliar o perfil sociodemográfico da população (Tabela 1), evidenciou-se predomínio do sexo feminino (89\%) e idade maior que 50 anos (36\%). Quanto ao cargo, identificou-se que $86 \%$ ocupam cargo assistencial e $14 \%$ de chefia. Quase metade da amostra (49\%) afirmou possuir empregos paralelos em outras instituições, sendo a maioria como enfermeiro (67\%). A maioria dos profissionais está formada há mais de 16 anos (74\%), e a média de tempo de trabalho na unidade foi de $15 \pm 11,68$. 
Tabela 1

Perfil Sociodemográfico dos Participantes

\begin{tabular}{lcccc}
\hline Variáveis & Categorias & N & $(\%)$ & \\
\hline Sexo & Fem & 62 & 89 & - \\
Faixa etária & Masc & 8 & 11 & - \\
& 20 a 30 & 4 & 6 & - \\
& 31 a 40 & 21 & 30 & - \\
& 41 a 50 & 20 & 29 & - \\
Cargo & mais de 50 & 25 & 36 & - \\
\multirow{3}{*}{ Outro trabalho } & Assistencial & 60 & 86 & - \\
& Chefia & 10 & 14 & - \\
Cargo do outro trabalho & Não & 36 & 51 & - \\
& Sim & 34 & 49 & - \\
& Chefe de setor & 2 & 6 & - \\
Tempo de formado & Enfermeiro & 23 & 67 & - \\
& Professor & 4 & 12 & - \\
& Supervisor de enfermagem & 1 & 3 & - \\
& Técnico de enfermagem & 4 & 12 & - \\
& 5 a 10 anos & 5 & 7 & - \\
& 11 a 15 anos & 13 & 19 & - \\
& mais de 16 anos & 52 & 74 & - \\
& & & & $15 \pm 11,68$ \\
\hline
\end{tabular}

As médias de estresse entre os enfermeiros variaram entre 1,0 e 6,0, sendo 7,0 a pontuação máxima. Conforme a Tabela 2, a maior parte (67\%) apresentou nível médio de estresse, $30 \%$ baixo nível de estresse e $3 \%$ alto nível de estresse. Destaca-se que 2 enfermeiros, 1 do $\mathrm{CTI}$ e 1 da emergência tiveram alto nível de estresse.

Tabela 2

Nivel de Estresse dos Enfermeiros do Hospital Universitário Clementino Fraga Filho

\begin{tabular}{lcc}
\hline Nível de estresse & $\mathrm{n}$ & $\%$ \\
\hline Baixo nível de estresse & 21 & 30 \\
Médio nível de estresse & 47 & 67 \\
Alto nível de estresse & 2 & 3 \\
\hline
\end{tabular}

Em seguida, analisou-se o nível de estresse em cada domínio proposto pela escala. Com relação aos enfermeiros do hospital de forma geral, todos os domínios foram avaliados como médio nível de estresse (entre 3,1 e 5,9). Conforme exposto na Figura 1, o domínio 
B (atividades relacionadas ao funcionamento adequado da unidade) teve a maior média de escore $(5,2)$. Dentre os itens deste domínio, destaca-se o item 2 (Reposição de material) como o mais desgastante (escore médio: 6,5).

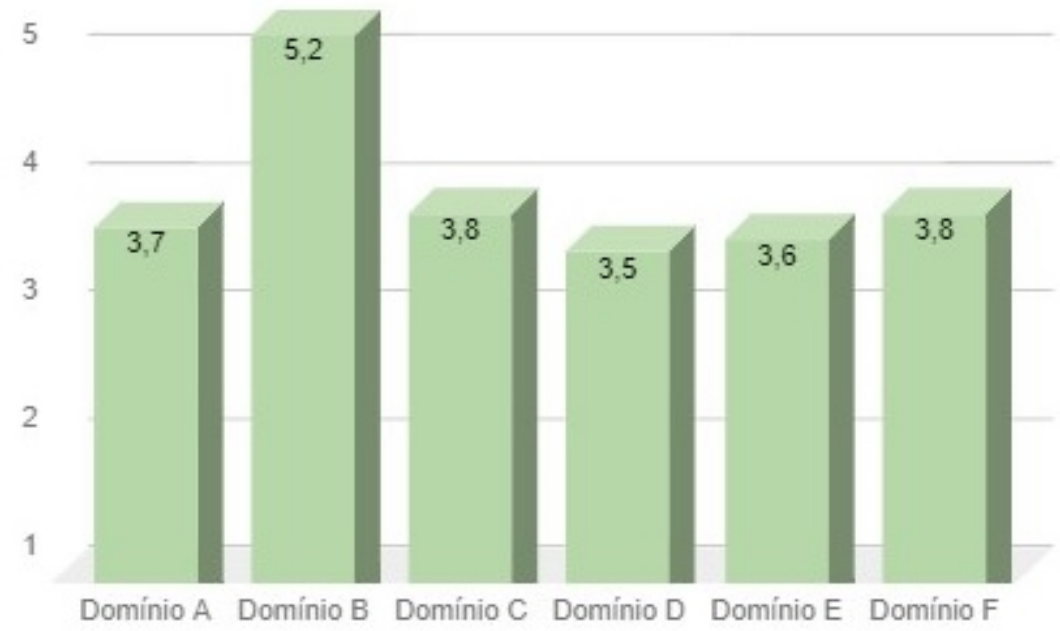

Figura 1. Média do nível de estresse por domínio para a amostra total.

Ao analisar os dados dos domínios dos enfermeiros por unidade de trabalho, observou-se que o domínio B (atividades relacionadas ao funcionamento adequado da unidade) obteve a maior média de escore de estresse em todas as unidades (Figura 2).

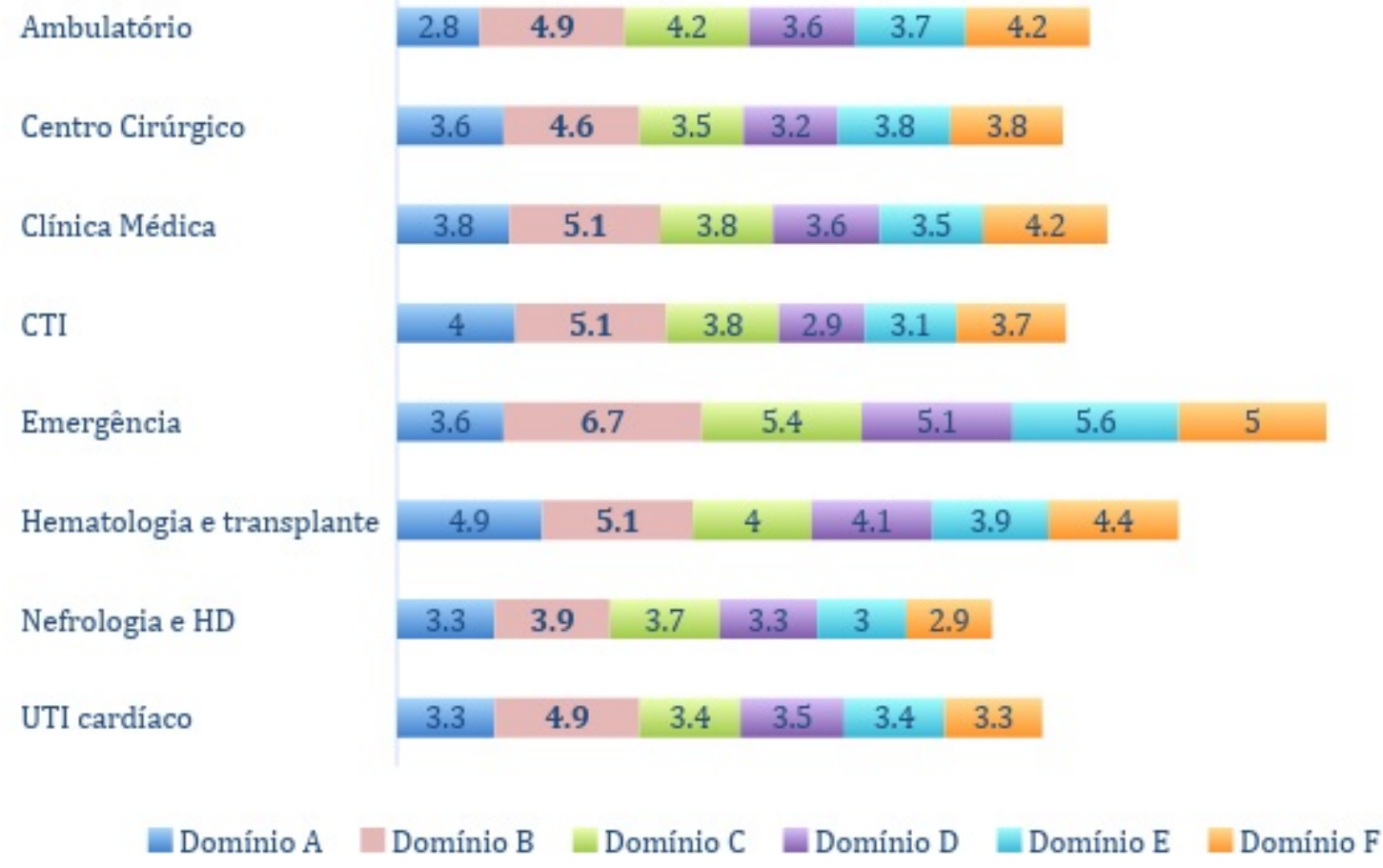

Figura 2. Média do nível de estresse por domínio para cada unidade. 


\section{Análise das Correlações}

Pelo teste ANOVA (Tabela 3 ), observou-se que não há correlação entre nível de estresse e faixa etária $(p=0,37)$, nível de estresse e tempo de formado $(p=0,36)$, e que não há diferença significativa entre as médias de estresse e as diferentes unidades $(p=0,24)$. Não foi encontrada correlação entre outro trabalho em paralelo com o do hospital estudado e o nível de estresse (teste t de student $p=0,87$ ) e entre este e o tempo de trabalho na unidade (coeficiente de correlação de pearson $p=-0,190)$. Destaca-se que a média do nível de estresse dos enfermeiros que exercem função de chefia é significantemente maior do que a média dos que não exercem (teste t de student $p=0,002$ ).

Tabela 3

Correlação entre as Variáveis da Amostra e o Nível de Estresse

\begin{tabular}{|c|c|c|}
\hline \multirow{2}{*}{ Variável } & \multicolumn{2}{|c|}{ Nível de estresse (Teste ANOVA) } \\
\hline & $F$ & Valor $p$ \\
\hline Faixa etária & 1,04 & 0,377 \\
\hline Tempo de formado & 1,01 & 0,368 \\
\hline \multirow[t]{3}{*}{ Unidades de trabalho } & 1,34 & 0,240 \\
\hline & \multicolumn{2}{|c|}{ Nível de estresse (Teste $t$ de student) } \\
\hline & $t$ & Valor $p$ \\
\hline $\begin{array}{l}\text { Possui outro trabalho } \\
\text { Não possui outro } \\
\text { trabalho }\end{array}$ & $-1,16$ & 0,875 \\
\hline \multirow{4}{*}{$\begin{array}{l}\text { Exercem função de } \\
\text { chefia } \\
\text { Não exercem função de } \\
\text { chefia }\end{array}$} & 2,90 & $0,0025^{*}$ \\
\hline & Níve & ciente de correlação $r$ de \\
\hline & & son) \\
\hline & & Valor $p$ \\
\hline $\begin{array}{l}\text { Tempo de trabalho na } \\
\text { unidade }\end{array}$ & & $-0,1902$ \\
\hline
\end{tabular}

\section{Análise Qualitativa}

Foi realizada a análise de conteúdo de Bardin para a pergunta aberta: "Quais ações você acha que poderiam amenizar o estresse do enfermeiro?". Os dados analisados foram agrupados em categorias de 
acordo com a regularidade com que os elementos apareceram. Assim, criaram-se 15 categorias, que foram alocadas em 6 áreas temáticas, conforme explícito na Figura 3.

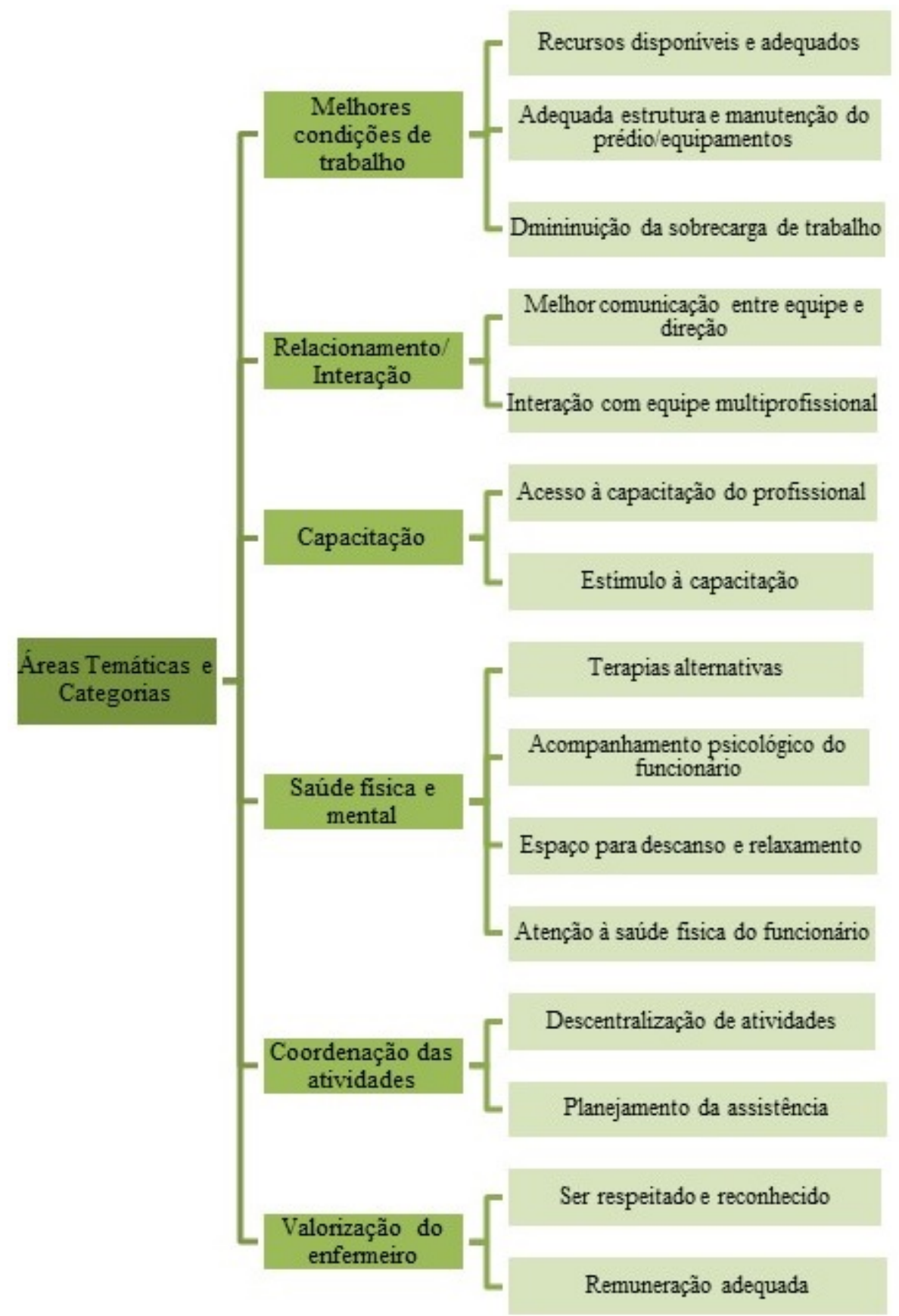

Figura 3. Categorias de análise da pergunta aberta "Quais ações você acha que poderiam amenizar o estresse?"

A área temática "melhores condições de trabalho" foi a mais citada como sugestão para amenizar o estresse (52\%). "Recursos disponíveis e adequados" foi a categoria mais manifestada pelos enfermeiros (32\%), seguida da categoria "Adequada estrutura e manutenção prédio/equipamentos (18\%). A segunda área temática 
mais citada foi "Relacionamento/Interação" (14\%), sendo a categoria melhor comunicação entre equipe e direção a mais apontada (10\%). Pela perspectiva dos enfermeiros, o cuidado com a saúde mental e física $(13 \%)$, tais como terapias alternativas $(6 \%)$, foi considerado como possibilidade para amenizar o estresse, assim como ter a oportunidade e estímulo para a capacitação profissional (9\%). Surgiram ainda ações relacionadas à coordenação das atividades do enfermeiro (8\%), como a descentralização de atividades administrativas $(4 \%)$ e ações referentes à valorização do enfermeiro $(4 \%)$.

\section{Discussão e Considerações Finais}

A análise dos resultados revelou que houve o predomínio feminino ( $89 \%$ ) na amostra estudada, resultado que condiz com estudos que avaliaram as características sociodemográficas de enfermeiros do Brasil (Fernandes, Portela, Griep, \& Rotenberg, 2017; Machado et al., 2016). Já a predominância da faixa etária de mais de 50 anos diferiu dos outros estudos que tiveram como resultado a predominância de idade entre 26 a 35 anos (Machado et al., 2016).

A maioria dos enfermeiros (67\%) apresentou nível médio de estresse. Outras pesquisas encontraram resultados similares (Kirhhof, Oshôa, Bublitz, Lopes, \& Squilavenato, 2016; Ratochinski, Powlowytsch, Grzelczak, Souza, \& Mascarenhas, 2016; Simonetti \& Bianchi, 2016). Destaca-se que quase a metade (43\%) dos participantes que obtiveram nível médio de estresse tiveram a pontuação entre 4,1 e 5,9 e, por isso, são considerados por diversos autores e pela autora da escala como "em grau de alerta" para o alto nível de estresse. Considera-se que o nível médio a alto de estresse pode trazer consequências negativas. Como apresentado anteriormente, essas consequências podem ser de vários tipos, como disfunções orgânicas e transtornos psicológicos, visto que o estresse é um dos principais fatores ambientais que predispõem à depressão e à diminuição da produtividade (Paulino, Prezotto, \& Calixto, 2015).

Não foi encontrada diferença significativa entre as médias de estresse das unidades. $\mathrm{Na}$ realidade do $\mathrm{HU}$ as especificidades dos setores parecem não ter sido suficientes para determinar uma diferença entre o nível de estresse. Outra suposição é a de que os itens mais indicados pelos enfermeiros como estressantes foram problemas relacionados ao funcionamento adequado da unidade como previsão e reposição de material, fatores comuns a todas as unidades. Apesar de não ter sido encontrada diferença estatística, destaca-se que os 2 enfermeiros considerados pela escala com alto nível de estresse são da emergência e do CTI. Enfermeiros que atuam nesses locais lidam constantemente com pacientes de risco, sofrimento dos familiares e 
rotina acelerada (Brochado \& Ribas, 2019; Soares, Medonça, Silva, \& Lanna, 2018).

Não foi identificada correlação entre as variáveis nível de estresse e tempo de trabalho, embora, de acordo com a literatura, o maior tempo de trabalho esteja relacionado a níveis mais baixos de estresse, em razão da propensão ao desenvolvimento de maior segurança técnica e de controle sobre as situações pelo enfermeiro ao longo do tempo (Guerrer \& Bianchi, 2008). Pode-se sugerir que esse resultado tenha ocorrido devido à não relação entre tempo de trabalho e controle das situações referentes aos estressores mais apontados como gerador de estresse (Domínio B: previsão, controle, levantamento, reposição de material, solicitação de revisão e consertos de equipamentos), pois estes se mostram mais externos ao profissional.

Quase metade da amostra (49\%) afirmou possuir outro vínculo empregatício. Apesar de não encontrar neste estudo correlação entre essa variável e o estresse, outras pesquisas demonstram que ter mais de um trabalho em paralelo gera sintomas relacionados ao estresse (Santos, Cavalcanti, Ataídes, \& Silva, 2018). É possível que, na presente amostra, o fato de trabalharem em outros locais não faça muita diferença devido ao tempo de formado, podendo contribuir para a adaptação a esse esquema de trabalho.

Destaca-se que a média do nível de estresse dos enfermeiros que exercem função de chefia é significantemente maior do que a média dos que não exercem. Outros estudos confirmam esse resultado (Guerrer \& Bianchi, 2008; Menzani \& Bianchi, 2009). Além disso, uma pesquisa que avaliou as condições de trabalho e o estresse em enfermeiros com cargos de chefia indicou que os que estão envolvidos nas atividades gerenciais possuem seis vezes mais chances de apresentar altos níveis de estresse comparado aos enfermeiros que atuam somente na assistência. A função de chefia demanda responsabilidade quanto à supervisão dos subordinados, que se soma a outras atividades do cargo, muitas vezes, administrativas, para as quais nem sempre o profissional foi preparado (Lindholm, 2006).

Com relação à avaliação dos estressores, o domínio B (atividades relacionadas ao funcionamento adequado da unidade) obteve a média de escore de estresse mais alta $(5,2)$ com relação aos enfermeiros de uma forma geral. Ao analisar cada unidade, o mesmo domínio (B) também foi considerado como o mais estressante por todas elas. Esses dados evidenciam que as atividades de previsão e reposição de material, controle de material e equipamento, solicitação de revisão e consertos de equipamentos e levantamento de quantidade de material existente na unidade são os fatores que mais geram estresse para os enfermeiros do HUCFF. Dentre esses, destaca-se o item "reposição de material" considerado o mais desgastante para o 
profissional. Tal fato já era esperado pelas condições da saúde pública, especificamente dos hospitais universitários do Rio de Janeiro na atualidade. Desta forma, não há como discutir esses resultados sem considerar o contexto sociopolítico econômico que vivenciamos no momento desta pesquisa. Como discutem Andrade, Michel, Adriano, Motta e Neto (2018), a realidade dos Hospitais Universitários tem sido caracterizada, há anos, pela deterioração causada pelo subfinanciamento e abandono pelo governo federal, refletida na precariedade de instalações físicas, recursos humanos e materiais.

De acordo com a análise qualitativa, a área temática “Melhores condições para o trabalho" foi a mais citada como sugestão para amenizar o estresse, tendo como categorias mais referenciadas: "Recursos disponíveis e adequados" e "Adequada estrutura e manutenção prédios/equipamentos". Esse resultado está de acordo com os resultados quantitativos.

A segunda área temática mais citada foi a "Relacionamento/Interação" (14\%), sendo a categoria "Melhor comunicação entre equipe e direção" a mais apontada (10\%), seguida da "Interação com equipe multiprofissional" (4\%). A comunicação entre chefia e os membros da equipe é considerado um fator desencadeante do estresse e também exerce influência na segurança do paciente, visto que a falta de comunicação ou comunicação ineficaz gera um ambiente favorável ao erro (Moraes Filho, Silva, \& Almeida, 2018; Henriques, Costa, \& Lacerda, 2016)

Outra sugestão para amenizar o estresse, dentro da área temática "saúde física e mental", foi a categoria "ter possibilidade de realizar terapias alternativas", também denominada terapias integrativas, como a acupuntura e shiatsu. A possibilidade de ter acompanhamento psicológico, também categorizado na área temática "saúde física e mental", foi apontada por 4 enfermeiros. Este item foi percebido pelos enfermeiros de outro estudo como primordial para auxiliar no reconhecimento de limites e compartilhar situações desgastantes vivenciadas no trabalho (Kessler \& Krug, 2012). A baixa frequência de sugestão dessa ação pelos enfermeiros desse estudo pode indicar que o atendimento psicológico aos trabalhadores não está difundido ou é insuficiente para a demanda existente, ou, ainda, pelo desconhecimento sobre a psicoterapia e seus benefícios pertinentes ao estresse.

Por fim, esse estudo avaliou a percepção do estresse e dos estressores de enfermeiros de um hospital universitário, comparou a percepção de estresse e de estressores dos enfermeiros de acordo com a unidade de saúde e coletou sugestões de ações para amenizar o estresse.

Os dados resultantes das análises quantitativas e qualitativas permitiram uma ampla compreensão sobre 0 estresse dos enfermeiros e os estressores. A predominância do nível médio de 
estresse configura-se uma realidade preocupante para a qualidade de vida e qualidade da assistência ao paciente, comprovando que os enfermeiros do HUCFF estão vulneráveis às consequências negativas do estresse.

As sugestões de ações coletadas revelam, assim como os resultados da escala utilizada, que a situação atual de precarização do hospital está relacionada com o estresse dos enfermeiros, fato que evidencia a importância desse estudo no momento atual, de forma a fornecer dados e sugestões para amenizar o desgaste causado por situações complexas que envolvem a crise econômica, a administração do hospital e políticas de saúde pública.

Sugere-se que sejam diagnosticados os fatores que estão dificultando a comunicação e interação na instituição. A promoção de momentos para discussão de casos, reuniões e espaços de escuta das necessidades do enfermeiro pode-se constituir em ações a serem implementadas.

Entende-se que, pela situação econômica e política, torna-se difícil concretizar todas as ações referidas pelos enfermeiros. No entanto, este estudo pode servir para mostrar o que mais está desgastando o enfermeiro e o que pode ser feito, mesmo nas condições atuais. A disponibilidade de acompanhamento psicológico, por exemplo, mostra-se fundamental para a saúde psicológica do profissional, e divulgar o serviço de psicologia ao trabalhador do HUCFF e facilitar o seu acesso constitui uma ação importante para a saúde do enfermeiro.

Algumas limitações deste estudo dizem respeito ao instrumento utilizado, que enfoca estressores relacionados somente às questões laborais, não abrangendo outras áreas da vida do enfermeiro, como a área social, familiar e da saúde, o que diminui a compreensão do estresse e dos estressores de forma mais ampla, além do fato de que as respostas dadas dependem do momento, condições físicas e emocionais, em que o profissional se encontra e da sua percepção, fatores inerentes a pesquisas com seres humanos.

Assim, propõe-se que estudos posteriores incluam na avaliação do estresse de enfermeiros elementos da vida social como a religião e relações familiares, já que esses fatores podem influenciar na vida profissional e, consequentemente, no estresse ocupacional. Sugerese também que estudos em outras instituições sejam realizados para verificação das diferenças encontradas em contextos diversos de modo a subsidiar a elaboração de estratégias voltadas para a saúde do profissional de enfermagem de um modo geral. 


\section{Referências}

Almeida, A. M. D. O., Lima, A. K. G., Vasconcelos, M. G. F., Lima, A. C. S., \& Oliveira, G. Y. M. D. (2016). Estresse ocupacional em enfermeiros que atuam em cuidados ao paciente crítico. Revista de enfermagem UFPE online, 10(5), 1663-1671. doi: 10.5205/1981-8963-v10i5a13541p1663-1671-2016.

Andrade, E. I. G., Michel, J., Adriano, E. R., Motta, J., \& Vecina Neto, G. (2018). Diferentes estratégias de gestão de estabelecimentos de saúde vinculados ao Sistema único de Saúde (SUS). Revista Debates GVsaúde, 0(esp), 40-57. Recuperado de http://bibliotecadigital.fgv.br/ojs/index.php/debatesgvsaude/art icle/ view/76844/73680

Bardin, L. (2011). Análise de conteúdo. Lisboa: Ediçoes, 70.

Bianchi, E. R. F. (2009). Bianchi Stress Questionnaire. Revista da Escola de Enfermagem da USP, 43(spe), 1055-1062. doi: $10.1590 /$ S0080-62342009000500009

Brasil, Ministério da Educação. (n.d.). Hospitais universitários. Recuperado de http: //portal.mec.gov.br/hospitais-universitarios Brochado, C., \& Ribas, J. L. C. (2019). Estresse Da Equipe De Enfermagem Na UTI. Revista Saúde e Desenvolvimento, 12(13), 44-57. Recuperado de: https://www.uninter.com/revistasaude/index.php/saudeDesenv olvimento/article/view/998

Fernandes, J. D. C., Portela, L. F., Griep, R. H., \& Rotenberg, L. (2017). Jornada de trabalho e saúde em enfermeiros de hospitais públicos segundo o gênero. Revista de saude publica, 51, 63. doi: 10.1590/S1518-8787.2017051006808

Guerrer, F. J. L., \& Bianchi, E. R. F. (2008). Caracterização do estresse nos enfermeiros de unidades de terapia intensiva. Revista da Escola de Enfermagem da USP, 42(2), 355-362. Recuperado http: // www. redalyc.org/articulo.oa? id =361033330020

Guido, L. A., Linch, G. F. C., Pitthan, L. O., \& Umann, J. (2011). Estresse, coping e estado de saúde entre enfermeiros hospitalares. Revista da Escola de Enfermagem da USP, 45(6), 1434-1439. doi: 10.1590/S0080-62342011000600022

Henriques, A. A. B., \& Costa, S. S., \& Lacerda, J. S. (2016). Assistência de enfermagem na segurança do paciente cirúrgico: Revisão integrativa. Cogitare Enfermagem, 21(4). Recuperado de http: //www.redalyc.org/articulo.oa?id=483653833023

International Labour Organization [ILO]. (2016). Workplace stress: A collective challenge. Suíça: International Labour Office. Recuperado de http://www.ilo.org/addisababa/mediacentre/news/WCMS_477712/lang--en/index.htm 
Kessler, A. I., \& Krug, S. B. F. (2012). Do prazer ao sofrimento no trabalho da enfermagem: o discurso dos trabalhadores. Revista Gaúcha de Enfermagem, 33(1), 49-55. doi:10.1590/S198314472012000100007

Kirhhof, R. S., Oshôa, L. M., Bublitz, S., Lopes, L. F. D., \& Squiavenato, M. C. A. (2016). Nível de estresse entre enfermeiros de um hospital filantrópico de médio porte. Revista de Enfermagem da UFSM, 6(1), 29-39. doi: $10.5902 / 2179769217829$

Lindholm, M. (2006). Working conditions, psychosocial resources and work stress in nurses and physicians in chief managers' positions. Journal of nursing management, 14(4), 300-309. doi: 10.1111/j.1365-2934.2006.00636.x

Lipp, M. E. N. (2017). O Treino de Controle do Estresse em grupo: um modelo da TCC. In C. B. Neufeld, \& B. P. Rangé, (Orgs.), Terapia cognitivo-comportamental em grupo: das evidências à prática (pp. 326-338). Porto Alegre, RS: Artmed.

Machado, M. H., Aguiar Filho, W., Lacerda, W. F., Oliveira, E., Lemos, W., Wermelinger, M., Vieira, M., Santos, M. R., Souza J unior, P. B., \& Barbosa, C. (2016). Características gerais da enfermagem: o perfil sócio demográfico. Enfermagem em Foco, 7(esp), 9-14. doi: 10.21675/2357-707X.2016.v7.nESP.686

Mauro, M. Y. C., Paz, A. F. D., Mauro, C. C. C., Pinheiro, M. A. D. S., \& Silva, V. G. (2010). Condições de trabalho da enfermagem nas enfermarias de um hospital universitário. Escola Anna Nery Revista de Enfermagem, 14(2), 244-52. Recuperado de http://eean.edu.br/

Médici, A. C. (2001). Hospitais universitários: passado, presente e futuro. Revista da Associação Médica Brasileira, 47(2), 149-56. doi: 10.1590/S0104-42302001000200034

Menzani, G., \& Bianchi, E. R. F. (2009). Stress dos enfermeiros de pronto socorro dos hospitais brasileiros. Revista eletrônica de Enfermagem, 11(2). doi: 10.5216/ree.v11.46978

Moraes Filho, I. M., \& Almeida, R. J. (2016). Estresse ocupacional no trabalho em enfermagem no Brasil: uma revisão integrativa. Revista Brasileira em Promoção da Saúde, 29(3), 447-454. doi: 10.5020/18061230.2016.p447

Moraes Filho, I. M., Silva, A. M. T. C., \& Almeida, R. J. (2018). Avaliação do estresse ocupacional de enfermeiros da estratégia saúde da família. Revista Eletrônica Gestão \& Saúde, 9(3), 335343. Recuperado http://ojs. bce.unb.br/index.php/rgs/article/view/20288

Oliveira, E. B., Gallasch, C. H., Silva Junior, P. P. A., Oliveira, A. V. R., Valério, R. L., \& Dias, L. B. S. (2017). Estresse ocupacional e burnout em enfermeiros de um serviço de emergência: a 
organização do trabalho. Revista Enfermagem UERJ, 25, 1-7. doi: 10.12957/reuerj. 2017.28842

Paschoal, T., \& Tamayo, A. (2004). Validação da escala de estresse no trabalho. Estudos de Psicologia, 9(1), 45-52. doi: 10.1590/S1413-294X2004000100006

Paulino, C. A., Prezotto, A. O., \& Calixto, R. F. (2015). Associação entre estresse, depressão e tontura: uma breve revisão. Revista Equilíbrio Corporal e Saúde, 1(1), 33-45. doi: 10.17921/2176-9524.2009v1n1p\%25p

Puerto, J. C., Soler, L. M., Montesinos, M. J. L., Marcos, A. P., \& Chorda, V. M. G. (2017). Uma nova contribuição para a classificação dos fatores estressores que afetam os profissionais de enfermagem. Revista Latino-Americana de Enfermagem, 25, 1-7. doi: 10.1590/1518-8345.1240.2895

Ratochinski, C. M. W., Powlowytsch, P. W. M., Grzelczak, M. T., Souza, W. C., \& Mascarenhas, L. P. G. (2016). O estresse em profissionais de enfermagem: uma revisão sistemática. Revista Brasileira de Ciências da Saúde, 20(4), 341-346. doi: 10.4034/RBCS.2016.20.04.12

Rodrigues, A. L., Barrichello, A., \& Morin, E. M. (2016). Os sentidos do trabalho para profissionais de enfermagem: um estudo multimétodos. Revista de Administração de Empresas, 56(2), 192-208. doi: 10.1590/S0034-759020160206

Santos, L. C., Cavalcanti, O. E., Ataídes, J. S., \& Silva, D. T. (2018). Fatores predisponentes à síndrome de burnout e estresse em enfermeiros na unidade de terapia intensiva. Revista de Enfermagem da Faciplac, 2(2), 1-12. Recuperado de http://revista.faciplac. edu.br/index. php/REFACl/article/view/57 $8 / 213$

Selye, H. (1965). The Stress Syndrome. AJ N The American Journal of Nursing, 65(3), 97-99. doi: 10.2307/3453119

Silva, A. H., \& Fossá, M. I. T. (2017). Análise de conteúdo: exemplo de aplicação da técnica para análise de dados qualitativos. Dados em Big Data, 1(1), 23-42. doi: 10.18391/qualitas.v16i1.2113

Simonetti, S. H., \& Bianchi, E. R. F. (2016). Estresse do enfermeiro que atua em unidade de internação. Revista de enfermagem UFPE online-ISSN: 1981-8963, 10(12), 4539-4546. doi: 10.5205/1981-8963-v10i12a11521p4539-4546-2016

Soares, J., Medonça, A., Silva, M., \& Lanna, M. (2018). O psicólogo no centro de terapia intensiva: relato de uma prática investigativa. Sinapse Múltipla, 7(2), 202-205. Recuperado de http://periodicos. pucminas. br/index. php/sinapsemultipla/article

Soratto, M. T., de Souza, M. P., Mattos, S. B., Ceretta, L. B., Gomes, K. M., \& Correa, S. M. (2016). O estresse da equipe de enfermagem no centro cirúrgico. Revista Interdisciplinar de 
Estudos em Saúde, 5(1), 179-192. Recuperado de http:// periodicos.uniarp.edu.br

Ueno, L. G. S., Bobroff, M. C. C., Martins, J. T., Machado, R. C. B. R., Linares, P. G., \& Gaspar, S. D. G. (2017). Estresse ocupacional: estressores referidos pela equipe de enfermagem. Revista de enfermagem UFPE, 11(4), 1632-8. doi:10.5205/reuol.976385423-1-SM.1104201710

Xavier, L. P., Reis, P. P. F., \& Frassão, M. C. G. O. (2016). O Trabalho do Psicólogo Junto à Equipe de Saúde. Revista Ciências em Saúde, 6(1), 66-77. doi: 10.21876/rcsfmit.v6i1.455

\author{
Endereço para correspondência \\ Jéssica Martins da Silva \\ Universidade Federal do Rio de Janeiro \\ Rua Rodolpho Paulo Rocco, 255, CAE - 12ㅇandar, Ilha do Fundão, CEP 21941-590, \\ Rio de Janeiro - RJ, Brasil \\ Endereço eletrônico: jessicamspsi@gmail.com
}

Lucia Emmanoel Novaes Malagris

Universidade Federal do Rio de J aneiro

Av. Pasteur, 250, Praia Vermelha, CEP 22290-240, Rio de Janeiro - RJ, Brasil

Endereço eletrônico: lucianovaes@terra.com.br

Recebido em: 16/10/2018

Reformulado em: 10/03/2019

Aceito em: 20/03/2019

\title{
Notas
}

* Pós-graduada em Psicologia Hospitalar - Clínica Médica pelo Hospital Universitário Clementino Fraga Filho (HUCFF/UFRJ).

** Doutora pela UERJ e Professora do Programa de Pós-Graduação em Psicologia do Instituto de Psicologia da UFRJ .

Este artigo de revista Estudos e Pesquisas em Psicologia é licenciado sob uma Licença Creative Commons Atribuição-Não Comercial 3.0 Não Adaptada. 